\title{
KRIGAGEM ORDINÁRIA E INVERSO DO QUADRADO DA DISTÂNCIA APLICADOS NA ESPACIALIZAÇÃO DE ATRIBUTOS QUÍMICOS DE UM ARGISSOLO
}

\section{ORDINARY KRIGING AND INVERSE-SQUARE-DISTANCE IN ESPACIALIZA- TION IN THE CHEMICAL ATTRIBUTES OF THE ULTISOL}

\author{
Gustavo Soares de SOUZA ${ }^{1}$ \\ Julião Soares de Souza LIMA ${ }^{2}$ \\ Alexandre Cândido XAVIER ${ }^{3}$ \\ Wadson Sebastião Duarte da ROCHA ${ }^{4}$
}

\begin{abstract}
RESUMO
Um dos fatores que influência a aplicação e utilização da agricultura de precisão é a acurácia com que os mapas de distribuição espacial dos atributos do solo são produzidos no processo de interpolação de dados. O objetivo deste trabalho foi comparar a eficiência da interpolação de dados por meio da krigagem ordinária (Kri) e do inverso do quadrado da distância (IQD) na estimativa de atributos químicos do solo. O experimento foi instalado na parte central de uma encosta, no município de Alegre (ES) sobre um Argissolo Vermelho-Amarelo, com textura argilosa, onde foi construída uma grade de amostragem de solo contendo 128 pontos, espaçados $10 \mathrm{~m}$, para análise dos atributos químicos $\mathrm{pH}, \mathrm{H}+\mathrm{Al}$ e SB . A análise geoestatística mostrou que todos os atributos químicos do solo apresentaram dependência espacial. Notou-se uma maior diferença visual entre os mapas interpolados por Kri e IQD para o atributo SB, apresentando o mapa interpolado por IQD uma maior variabilidade, resultado de uma menor uniformidade dos tons de cinza. Os métodos de interpolação foram eficientes na inferência de dados para os três atributos químicos analisados. Os métodos de interpolação utilizados não apresentaram diferenças significativas, através da análise dos erros da estimativa.
\end{abstract}

Palavras chave: Krigagem ordinária; inverso do quadrado da distância; geoestatística.

\begin{abstract}
One of the factors that influence the implementation and use of precision agriculture is the accuracy with which the maps of spatial distribution of soil attributes are produced in the process of interpolation of data. The objective was to compare the efficiency of the interpolation of data through the ordinary kriging (Kri) and of the inverse square of the distance (ISD) in the estimate of chemical attributes in the soil. The experiment was installed in the central part of a hillside, in the municipality of Alegre (ES), on an Ultisol Red-Yellow, with loamy texture, where a grating of soil sampling was used containing 128 points, spaced $10 \mathrm{~m}$ for analysis of the attributes chemical $\mathrm{pH}, \mathrm{H}+\mathrm{Al}$ and $\mathrm{SB}$. The geostatistics analysis showed that all of the chemical attributes of the soil presented spatial dependence. It was noticed a larger difference look among the maps interpolated by Kri and ISD for the attribute SB, presenting the map interpolated by ISD a larger variability, result of a smaller uniformity of the ash tones. The interpolation methods were efficient in the inference of data for the three analyzed chemical attributes. The interpolation methods used didn't present significant differences, through the analysis of the mistakes of the estimate.

Key-words: Ordinary kriging; inverse-square-distance; geostatistics.
\end{abstract}

\footnotetext{
${ }^{1}$ Eng. Agrônomo, Doutorando em Engenharia Agrícola Faculdade de Engenharia Agrícola (FEAGRI)/ Universidade Estadual de Campinas (UNICAMP). Av. Candido Rondon, 501, CP 6011, CEP 13083-875, Campinas, São Paulo, Brasil. Bolsista CNPq. E-mail: gustavo.souza@feagri.unicamp.br. Autor para correspondência

2 Eng. Agrícola, D.Sc. Professor Departamento de Engenharia Rural Centro de Ciências Agrárias (CCA) / Universidade Federal do Espírito Santo (UFES). Alegre, Espírito Santo, Brasil. E-mail: limajss@yahoo.com.br

3 Eng. Agrícola, D.Sc. Professor Departamento de Engenharia Rural Centro de Ciências Agrárias (CCA) / Universidade Federal do Espírito Santo (UFES). Alegre, Espírito Santo, Brasil. E-mail: xavier@cca.ufes.br

${ }^{4}$ Eng. Agronômo, D.Sc. Pesquisador Empresa Brasileira de Pesquisa Agropecuária / Embrapa Gado de Leite (EMBRAPA/CNPGL). Juiz de Fora, Minas Gerais, Brasil. E-mail: wadson@cnpgl.embrapa.br
} 
SOUZA, G.S. et al. Krigagem ordinária e inverso do quadrado...

\section{INTRODUÇÃO}

Considerando a agricultura de precisão, um dos fatores limitantes de sua utilização é a precisão com que os mapas de distribuição espacial dos atributos do solo são produzidos no processo de interpolação de dados. O levantamento dos atributos químicos observados ao longo do campo e o posterior mapeamento dos solos permitem visualizar a variação de fertilidade dentro de uma área, permitindo obter dados para buscar produtividades elevadas, além da utilização mais racional dos insumos agrícolas e de um menor impacto da atividade agrícola no ambiente (Angelico \& Silva, 2006).

Estimar dados de atributos do solo é muito importante em diversos contextos, muitas vezes ligado a adoção do manejo agrícola diferenciado em áreas heterogêneas. Assim, a obtenção da correta distribuição espacial para tais atributos é relevante no planejamento agrícola, no que diz respeito à instalação e manejo das culturas (Silva et al., 2008). Da mesma forma, em áreas de pastagem, os mapas de atributos químicos podem ser utilizados para análise da variabilidade espacial da fertilidade do solo e identificação de zonas de manejo (Souza et al., 2008a; Souza et al., 2008b). Gomes et al. (2007) também utilizaram mapas de atributos do solo para análise ambiental em uma bacia hidrográfica com diferentes usos e manejos, visualizando fontes potenciais de degradação dos recursos ambientais.

Os mapas de distribuição espacial são gerados a partir de modelagem matemática por métodos de interpolação, ou seja, estimam-se valores para locais não amostrados, a partir de um determinado número de pontos observados em campo. No entanto, é necessário comparar os métodos de interpolação, pois os resultados obtidos na geração dos mapas poderão ser diferentes dependendo do método utilizado (Tieppo et al., 2007). Os métodos de interpolação usados tornamse, então, ferramentas imprescindiveis, pois os resultados gerados pelos diferentes métodos podem subestimar ou superestimar o valor do atributo em estudo (Couto et al., 2002). Nesse sentido, a qualidade da estimação depende tanto da escolha dos métodos de interpolação, como também da aplicação apropriada de métodos indicados para as características dos dados em estudo.

Entre os métodos de interpolação disponíveis na literatura, os utilizados com maior destaque são krigagem e inverso do quadrado da distância (Carvalho \& Assad, 2005; Silva et al., 2008). O método da krigagem ou geoestatístico usa a dependência espacial entre amostras vizinhas, expressa no semivariograma, para estimar valores em qualquer posição dentro do campo, sem tendência e com variância mínima, sendo assim um estimador ótimo (Carvalho \& Assad, 2005). Quando se trabalha considerando a dependência espacial, podem-se reduzir os erros aleatórios pelo controle de uma parcela deste erro associada à dependência espacial (Mello et al., 2003a).

No método do inverso do quadrado da distância o fator de ponderação, como o nome já indica, é o inverso do quadrado da distância euclidiana entre o ponto conhecido e o estimado. Esse método é considerado simples e de fácil aplicação, no entanto menos acurado que a krigagem, uma vez que não considera o padrão da estrutura da dependência espacial (Mello et al., 2003b; Silva et al., 2008). Entretanto, Couto et al. (2002) relataram que tanto a krigagem, como o inverso do quadrado da distância apresentam destaque de utilização na inferência de dados, sendo que o melhor depende principalmente do atributo a ser estimado.

Mello et al. (2003a), trabalhando com a estimativa de parâmetros da equação de chuvas intensas, constataram que a krigagem produziu menores erros médios comparativamente ao método do inverso do quadrado da distância. Carvalho \& Assad (2005) observaram que o interpolador geoestatístico apresentou melhor resultado que o inverso do quadrado da distância e que a curvatura mínima na espacialização da precipitação pluviométrica para o Estado de São Paulo, com menor quadrado médio do erro. Silva et al. (2008), estudando atributos químicos do solo, concluíram que a interpolação dos dados por krigagem apresentou uma melhor performace, visto que o inverso do quadrado da distância possuiu maior erro na estimação dos dados.

No entanto, o método do inverso do quadrado da distância também apresenta acurácia satisfatória, além de exigir uma análise mais simples, o que pode tornar o processo menos oneroso e, em alguns casos, proporciona resultados semelhantes à krigagem. Kanegae Júnior et al. (2006) observaram uma maior redução na variância média e no erro da amostragem com o inverso do quadrado da distância em relação a krigagem ordinária na estratificação de povoamentos de eucalipto.

Com base nesta premissa, este trabalho objetivou comparar a eficiência da interpolação de dados regionalizados por meio da krigagem ordinária e do inverso do quadrado da distância na estimativa de atributos químicos em um Argissolo Vermelho-Amarelo.

\section{MATERIAL E MÉTODOS}

A área de estudo localiza-se na Bacia Hidrográfica do Itapemirim, sub-bacia do Córrego Horizonte, na área da Escola Agrotécnica Federal de Alegre, situada no distrito de Rive, Município de Alegre, a sudoeste do estado do Espírito Santo. A área de estudo encontra-se nas coordenada geográfica $20^{\circ} 46^{\prime} 2,8^{\prime \prime}$ de latitude Sul e $41^{\circ} 27^{\prime}$ 39,2 " de longitude Oeste.

A região apresenta clima tropical (Aw), pelo sistema de Köppen, com duas estações bem definidas, sendo uma quente e chuvosa (outubro a abril) e outra fria e seca (maio a setembro), com temperatura média mensal de $23,9{ }^{\circ} \mathrm{C}$ e 
precipitação total anual média de $1.346 \mathrm{~mm}$. A área está localizada dentro do Bioma Mata Atlântica e sua vegetação natural constituiu-se de floresta estacional semidecidual. $O$ relevo da área em estudo é predominantemente acidentado, com declividade variando entre 30 a 45 graus. O solo da área em estudo foi caracterizado como Argissolo Vermelho-Amarelo Distrófico típico (PVAd), com textura argilosa apresentando os teores médios de areia, silte e argila iguais a 340, 223 e $434 \mathrm{~g} \mathrm{~kg}^{-1}$, respectivamente (EMBRAPA, 2006).

O experimento foi instalado na parte central de uma encosta (Figura 1), formando basicamente um compartimento homogêneo no local. A área experimental constou de dois tipos de uso do solo, apresentando no lado esquerdo do terreno ausência de cultivo com cobertura vegetal natural e no lado direto pastagem de Brachiaria decumbens, implantada no ano de 2000 em substituição a pastagem nativa, após preparo primário do solo com arado de aiveca de tração animal em nível e correção da acidez do solo com aplicação de calcário dolomítico somente na época de plantio. O sistema de pastejo é extensivo, com uma média anual de uma unidade animal por hectare, sendo uma maior concentração observada nos períodos de chuva, podendo chegar a três unidades por hectare e uma menor concentração no período de estiagem.

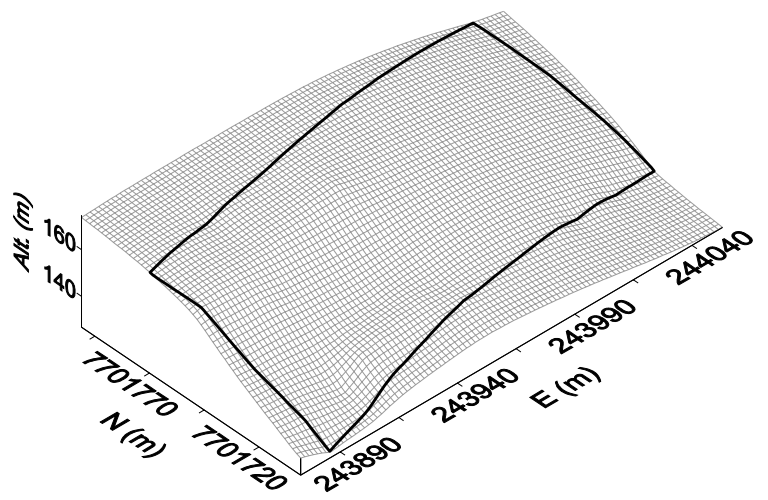

FIGURA 1 - Modelo digital de elevação contendo os limites da parcela experimental em estudo.

Foi utilizada uma grade regular de amostragem do solo contendo 128 pontos, espaçados $10 \mathrm{~m}$ e distribuídos em uma área de aproximadamente 1,12 ha. Para o processo de marcação dos pontos foram utilizados equipamentos de topografia e sistema de posicionamento global. O solo foi coletado em cada ponto de amostragem na profundidade de 0,00-0,20 $\mathrm{m}$ com auxílio de um trado tipo sonda para análise química.

Os atributos do solo a serem analisados foram: $\mathrm{pH}$ em água, acidez potencial $(\mathrm{H}+\mathrm{Al})$ e soma de cátions básicos (SB), conforme EMBRAPA (1997). As análises foram realizadas no Laboratório de Física e Química do Solo do Centro de Ciências Agrárias da Universidade Federal do Espírito Santo.

Os dados foram submetidos à análise descritiva, utilizando medidas de posição e dispersão. A normalidade dos dados foi verificada por meio do teste de Kolmogorov-Smirnov $(P<0,05)$. Em seguida, foi realizada a análise geoestatística para verificar a existência e, neste caso, quantificar o grau de dependência espacial dos atributos em estudo, a partir do ajuste de modelos teóricos aos semivariogramas experimentais isotrópicos estimados pela equação 1, descrita por Vieira (2000), utilizando o programa $\mathrm{GS}^{+}$(Robertson, 1998):

$$
\gamma^{*}(h)=\frac{1}{2 N(h)} \sum_{i=1}^{N(h)}\left[Z\left(x_{i}\right)-Z\left(x_{i}+h\right)\right]^{2}
$$

em que: ${ }^{*}$ é a semivariância estimada e $N(h)$ é o número de pares de valores medidos $Z\left(x_{i}\right)$ e $Z$ $\left(x_{i}+h\right)$ separados por um vetor $h$.

A presença de estacionaridade fraca ou hipótese intrínseca foi assumida, indicando a presença de variância finita no conjunto dos dados analisados. Os modelos teóricos testadas foram: esférico, exponencial e gaussiano. A escolha do modelo mais adequado foi baseada no maior coeficiente de determinação do semivariograma $\left(R^{2}\right)$ e na menor soma de quadrados do resíduo (SQR).

O ajuste dos modelos de semivariogramas permitiu definir os seguintes parâmetros: efeito pepita $\left(\mathrm{C}_{0}\right)$, patamar $\left(\mathrm{C}_{0}+\mathrm{C}\right)$, alcance $(\mathrm{a})$ e índice de dependência espacial (IDE). $O$ índice de dependência espacial representa uma relação em porcentagem do quanto à dependência espacial, quantificada pelo modelo de semivariograma, contribui para a variabilidade dos dados. O IDE foi classificado conforme critério adotado por Zimback (2001) em forte, médio e baixo, para os intervalos: IDE $>75 \%$, $25<$ IDE $\leq 75 \%$ e IDE $\leq 25 \%$, respectivamente. 
SOUZA, G.S. et al. Krigagem ordinária e inverso do quadrado...

Constatada a presença de dependência espacial entre os dados, foram realizadas inferências por krigagem ordinária (Kri) para locais não medidos a partir dos pontos amostrados. Essa técnica interpola valores com as condições de estimativa sem tendenciosidade e com desvios mínimos em relação aos valores conhecidos (Grego \& Vieira, 2005), considerando a estrutura de variabilidade espacial encontrada para o atributo, sendo definida pela equação 2

$\hat{Z}_{i}=\sum_{i=1}^{n} \lambda_{i}^{*} Z_{i}$

em que: $\hat{Z}_{i}$ é o valor interpolado; $\lambda_{i}$ é o peso atribuídos aos valores amostrados; $Z_{i}$ é o valor do atributo amostrado; $n$ é o número de localidades vizinhas empregadas para interpolação do ponto, sendo que o somatório dos pesos ${ }^{\lambda_{i}}$ deve ser igual a um.

O outro método utilizado neste trabalho foi o inverso do quadrado da distância (IQD), que é um interpolador determinístico univariado de médias ponderadas. De acordo com este método quanto mais distante um ponto observado estiver do estimado, menor será seu peso, ou seja, menor será sua influência sobre o valor de inferência. O IQD é definido pela equação 3:

$$
\hat{Z}_{i}=\frac{\sum_{i=1}^{n}\left(\frac{1}{d_{i}^{2}} * Z_{i}\right)}{\sum_{i=1}^{n}\left(\frac{1}{d_{i}^{2}}\right)}
$$

em que: $\hat{Z}_{i}$ é o valor interpolado; $Z_{i}$ é o valor do atributo amostrado; $d_{i}$ é a distância euclidiana entre o ponto amostrado e o estimado.

No presente estudo, trabalhou-se com 8 pontos amostrados mais próximos da localidade a ser interpolada para ambos os métodos, sendo que para a krigagem ordinária levou-se em consideração apenas os pontos que estivessem dentro do raio do alcance da dependência espacial obtido por cada atributo.

A avaliação da precisão dos interpoladores foi realizada através da análise das estatísticas: coeficiente de determinação da validação cruzada $\left(R^{2} v c\right)$, erro médio absoluto (EMA) e erro médio relativo (EMR). Os erros médios absoluto e relativo foram estimados através das equações 4 e 5, respectivamente:

$E M A=\frac{1}{n} * \sum_{i=1}^{n}\left|Z_{i}-\hat{Z}_{i}\right| * 100$
$E M R=\frac{1}{n} * \sum_{i=1}^{n} \frac{\left|Z_{i}-\hat{Z}_{i}\right|}{Z_{i}} * 100$

em que: EMA e EMR são, respectivamente, os erros médios absoluto e relativo dos $n$ pontos amostrados; $Z_{i}$ é o valor determinado na análise de solo do atributo no ponto amostrado; $\hat{Z}_{i}$ é o valor estimado pelo método de interpolação.

A significância do $R^{2}$ vc foi analisada através do teste $F(P<0,01)$, entre o valor observado e o estimado pelo método de interpolação. As estatísticas referentes aos erros da estimativa foram comparadas pelo teste t-Student $(P<0,05)$, permitindo analisar a ocorrência de diferença significativa entre os métodos de inferências.

\section{RESULTADOS E DISCUSSÃO}

$\mathrm{Na}$ Tabela 1, são apresentados os valores da estatística descritiva dos atributos químicos do solo. Os valores médios dos atributos químicos $\mathrm{pH}$, $\mathrm{H}+\mathrm{Al}$ e SB foram classificados em médio $(5,0-5,9)$, alto $\left(>5,0 \mathrm{cmol}_{\mathrm{c}} \mathrm{dm}^{-3}\right)$ e baixo $\left(<2,0 \mathrm{cmol}_{\mathrm{c}} \mathrm{dm}^{-3}\right)$ respectivamente, de acordo com o manual de recomendação de calagem e adubação para o Estado do Espírito Santo (Prezotti et al., 2007), indicando uma deficiência dos cátions nutrientes na área e um excesso de hidrogênio e alumínio, que prejudica o desenvolvimento do sistema radicular das plantas, bem como influencia na disponibilidade dos nutrientes.

A média e mediana apresentaram valores próximos, indicando uma tendência da distribuição normal dos dados, assim como valores de assimetria próximos de zero, no entanto a curtose apresentou valores negativos distantes de zero, o que representa uma maior dispersão dos dados em relação a distribuição normal. A realização do teste de normalidade confirmou que os atributos $\mathrm{H}+\mathrm{Al}$ e SB apresentaram distribuição normal pelo teste Kolmogorov-Smirnov $(\mathrm{P}<0,05)$, fato que não ocorreu para o $\mathrm{pH}$. Apesar da normalidade ser uma das pressuposições da estatística clássica, ela não é uma exigência da geoestatística. $\mathrm{Na}$ geoestatística, mais importante que a normalidade dos dados é a ocorrência ou não do efeito proporcional em que a média e a variância dos dados não sejam constantes na área de estudo (Souza et al., 2004).

Observou-se grande amplitude dos dados dos atributos em estudo, apresentando uma relação entre o valor máximo e mínimo de 1,32; 2,05 e 7,11 para os atributos $\mathrm{pH}, \mathrm{H}+\mathrm{Al}$ e $\mathrm{SB}$, respectivamente. Essa amplitude revelou os problemas que podem ocorrer quando se utiliza somente a média dos atributos do solo como base para análise e interpretação de resultados em experimentos de campo.

Observou-se que o atributo SB apresentou a maior variabilidade, com um coeficiente de 
SOUZA, G.S. et al. Krigagem ordinária e inverso do quadrado...

TABELA 1 - Estatística descritiva dos atributos químicos do solo $\mathrm{pH}, \mathrm{H}+\mathrm{Al}$ e soma de cátions básicos (SB).

\begin{tabular}{cccc}
\hline Atributo & $\begin{array}{c}\mathrm{pH} \\
\mathrm{em} \text { água }\end{array}$ & $\begin{array}{c}\mathrm{H}+\mathrm{Al} \\
\mathrm{cmol}_{\mathrm{c}} \mathrm{dm}^{-3}\end{array}$ & $\begin{array}{c}\mathrm{SB} \\
\mathrm{cmol}_{\mathrm{c}} \mathrm{dm}^{-3}\end{array}$ \\
\hline $\bar{x}$ & 5,08 & 5,27 & 1,63 \\
Md & 5,05 & 5,12 & 1,57 \\
Mín. & 4,40 & 3,55 & 0,46 \\
Max. & 5,80 & 7,26 & 3,27 \\
CV & 8,39 & 18,89 & 37,13 \\
$\mathrm{CS}_{\mathrm{S}}$ & 0,14 & 0,12 & 0,24 \\
$\mathrm{CK}_{\mathrm{K}}$ & $-1,40$ & $-1,10$ & $-0,55$ \\
$\mathrm{KS}$ & $*$ & $\mathrm{~ns}$ & $\mathrm{~ns}$ \\
\hline
\end{tabular}

$\bar{x}$

- média; Md - Mediana; Máx - máximo; Mín.- mínimo; CV- coeficiente de variação; $\mathrm{C}_{\mathrm{S}}$ - assimetria; $\mathrm{C}_{\mathrm{K}}$ - curtose; KS- teste de normalidade de Kolmogorov-Smirnov; * distribuição não normal $(P>0,05)$.

variação $(\mathrm{CV})$ superior a $30 \%$, enquanto que o $\mathrm{pH}$ apresentou o menor $\mathrm{CV}$. $\mathrm{O}$ pH do solo geralmente indica baixo $\mathrm{CV}$, uma vez que este apresenta escala logarítmica, dessa forma grandes variações da concentração hidrogeniônica na solução do solo, podem resultar em pequenas variações no valor de $\mathrm{pH}$.

A análise geoestatística, efetuada por meio do ajuste do modelo teórico ao semivariograma experimental, mostrou que os atributos químicos do solo apresentaram dependência espacial (Tabela 2). O modelo que melhor ajustou-se aos dados foi o gaussiano, apresentando $\mathrm{R}^{2}$ superior a $93 \%$ para todos os atributos e a menor SQR quando comparado aos demais modelos testados. De acordo com Robertson (1998), a SQR é um parâmetro mais robusto do que o $R^{2}$ e propicia uma medida mais exata do modelo que se ajusta aos dados, sendo os dois parâmetros usados na escolha do melhor modelo.

TABELA 2 - Análise geoestatística dos atributos químicos do solo pH, H+Al e soma de cátions básicos (SB).

\begin{tabular}{ccccccccc}
\hline Atributo & Modelo & $\mathrm{C}_{0}$ & $\mathrm{C}$ & $\mathrm{C}_{0}+\mathrm{C}$ & $\begin{array}{c}\mathrm{a} \\
(\mathrm{m})\end{array}$ & $\begin{array}{c}\text { IDE } \\
(\%)\end{array}$ & $\begin{array}{c}\mathrm{R}^{2} \\
(\%)\end{array}$ & $\mathrm{SQR}$ \\
\hline \multirow{3}{*}{$\mathrm{pH}$} & Esf & 0,001 & 0,335 & 0,336 & 156 & 99,7 & 90,5 & 0,031 \\
& Exp & 0,001 & 0,590 & 0,591 & 509 & 99,8 & 85,7 & 0,038 \\
& Gau & 0,001 & 0,347 & 0,348 & 128 & 99,7 & 93,3 & 0,016 \\
& Esf & 0,001 & 1,714 & 1,715 & 171 & 99,9 & 94,7 & 0,344 \\
$\mathrm{H}+\mathrm{Al}$ & Exp & 0,001 & 2,000 & 2,001 & 293 & 99,8 & 92,7 & 0,576 \\
& Gau & 0,003 & 1,721 & 1,724 & 133 & 99,8 & 97,3 & 0,136 \\
& Esf & 0,143 & 0,199 & 0,342 & 58 & 58,2 & 92,2 & 0,020 \\
& SB & 0,121 & 0,265 & 0,386 & 95 & 68,5 & 91,0 & 0,023 \\
& Gau & 0,174 & 0,176 & 0,350 & 53 & 50,1 & 93,2 & 0,018 \\
\hline
\end{tabular}

Esf- esférico; Exp- exponencial; Gau- gaussiano; $\mathrm{C}_{0^{-}}$efeito pepita; C- variância estrutural; $\mathrm{C}_{0}+\mathrm{C}$ - patamar; a- alcance; IDEíndice de dependência espacial; $R^{2}$ - coeficiente de determinação do semivariograma; SQR- soma de quadrados do resíduo.

$\mathrm{Na}$ Figura 2, são apresentados os modelos de semivariogramas escolhidos ajustados aos dados experimentais dos atributos químicos do solo. Observou-se que o ajuste do modelo permitiu construir semivariogramas com patamares bem definidos (Figuras 2A, 2B e 2C), indicando a presença de variância finita, desta forma, a hipótese intrínseca que é uma exigência mínima para a análise geoestatística foi atendida. 
SOUZA, G.S. et al. Krigagem ordinária e inverso do quadrado...
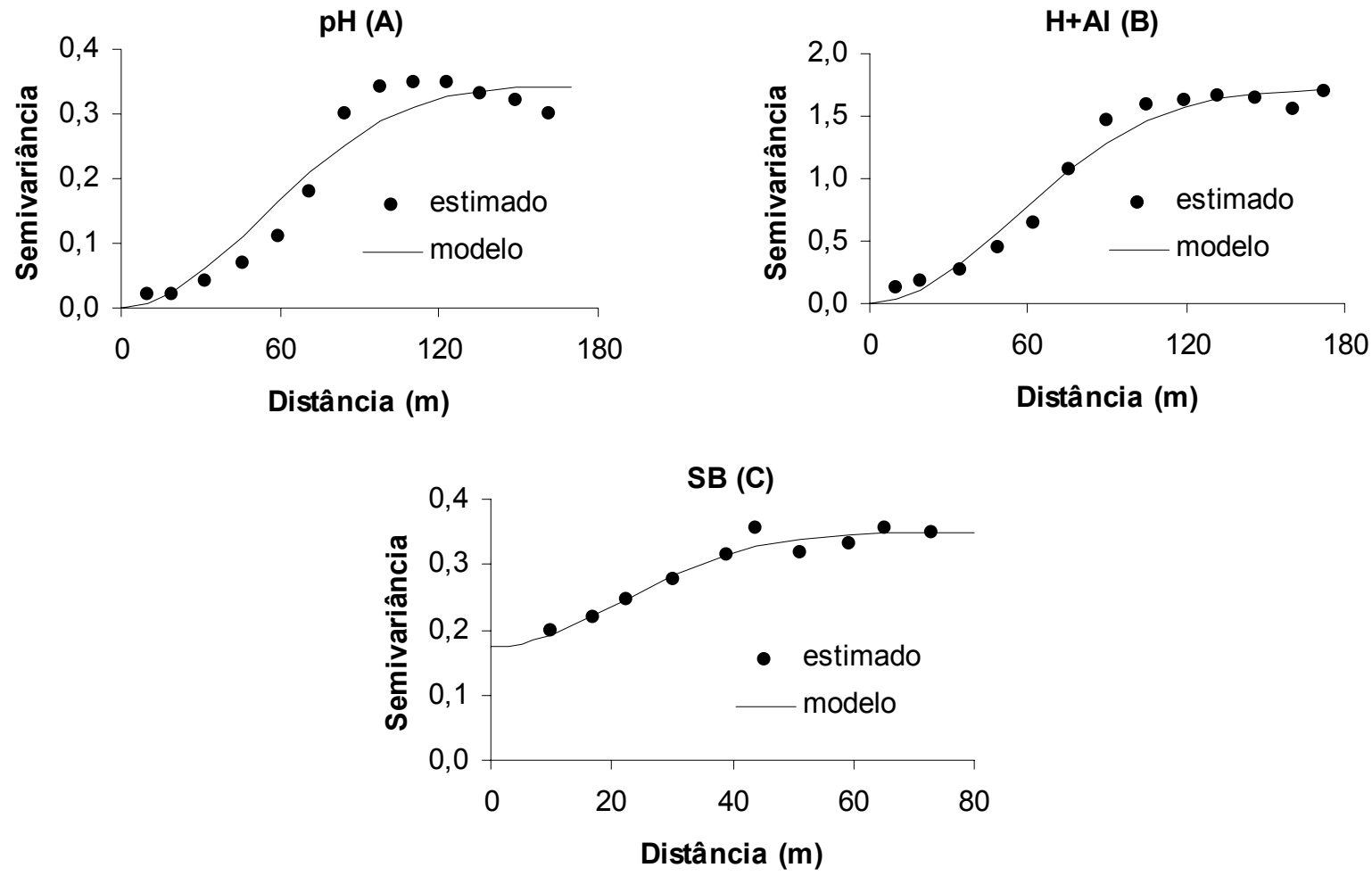

FIGURA 2 - Semivariogramas dos atributos químicos do solo $\mathrm{pH}(\mathrm{A}), \mathrm{H}+\mathrm{Al}(\mathrm{B})$ e soma de cátions básicos $(\mathrm{SB})(\mathrm{C})$ referentes a parcela em estudo.

Com relação ao alcance da dependência espacial, observou-se o menor valor para a SB (53 $\mathrm{m})$, enquanto $\mathrm{pH}$ e $\mathrm{H}+\mathrm{Al}$ apresentaram valores bem superiores (128 e $133 \mathrm{~m})$. O valor do alcance define a distância máxima até onde o valor de um atributo possui relação de dependência espacial com o seu próximo, assim determinações realizadas a distâncias menores que o alcance são correlacionadas, o que permite que se façam interpolações para espaçamentos menores que os amostrados (Silva et al., 2003).

De acordo com Corá et al. (2004), os valores de alcance podem influenciar na qualidade das estimativas, uma vez que ele determina o número de valores usados na interpolação, assim estimativas feitas com interpolação por krigagem ordinária utilizando valores de alcances maiores tendem a ser mais confiáveis, apresentando mapas que representem melhor a realidade.

O efeito pepita $\left(C_{0}\right)$ é a variância não explicada ou ao acaso e que não foi detectada na escala de amostragem, enquanto a variância estrutural $(C)$ representa a porção da variabilidade dos dados devido à dependência espacial e quantificada no ajuste do modelo. Dessa forma, quanto maior for à contribuição da $\mathrm{C}_{0}$ em relação ao patamar, maior será o erro e pior será a estimativa pela krigagem ordinária. Esses resultados podem ser visualizados mais claramente, através da análise do índice de dependência espacial (IDE), que indica a contribuição da dependência espacial (C) em relação à variabilidade dos dados $\left(\mathrm{C}_{0}+\mathrm{C}\right)$. Os atributos apresentaram IDE superior a $50 \%$, sendo classificada em forte dependência espacial para o $\mathrm{pH}$ e $\mathrm{H}+\mathrm{Al}$ e moderada para $\mathrm{SB}$, conforme Zimback (2001).

Na Figura 3, são apresentados os mapas interpolados por meio da krigagem ordinária (Kri), que considera a análise da estrutura da dependência espacial, e inverso do quadrado da distância (IQD).

Observou-se uma pequena diferença visual entre os mapas interpolados por Kri e IQD para os atributos químicos $\mathrm{pH}$ (Figuras $3 \mathrm{~A}$ e $3 \mathrm{~B}$ ) e $\mathrm{H}+\mathrm{Al}$ (Figuras $3 \mathrm{C}$ e 3D), apresentando uma maior concentração dos maiores valores de $\mathrm{pH}$ no lado direito da área, ocorrendo o inverso para o $\mathrm{H}+\mathrm{Al}$. Os níveis de $\mathrm{pH}$ do solo estão relacionados com os teores de $\mathrm{H}+\mathrm{Al}$, apresentando uma correlação significativa $(P<0,05)$ inversamente proporcional $\left(r=-0,85^{\star}\right)$. Isso ocorre porque o aumento do $\mathrm{pH}$ do solo proporciona uma menor solubilização do hidrogênio e alumínio presentes na solução do solo, sendo que em $\mathrm{pH}$ acima de 5,5 até 7,0 , a solubilização do alumínio é mínima (Sousa et al., 2007). Dessa forma, o aumento do $\mathrm{pH}$ na área de pastagem, proveniente da aplicação de calcário no período de implantação da gramínea, proporcionou a diminuição do $\mathrm{H}+\mathrm{Al}$ do solo. 
SOUZA, G.S. et al. Krigagem ordinária e inverso do quadrado...

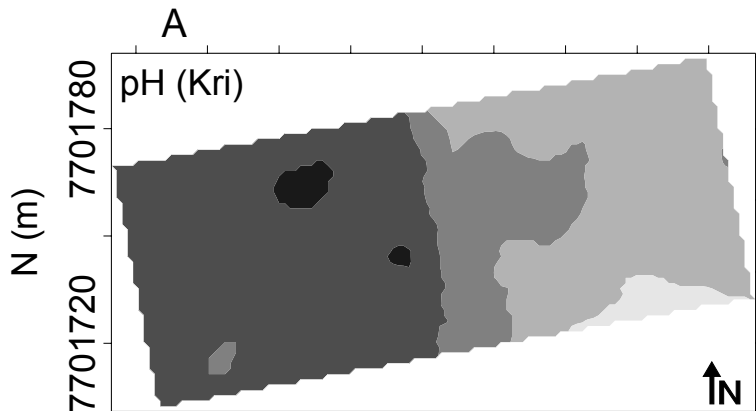

$B$

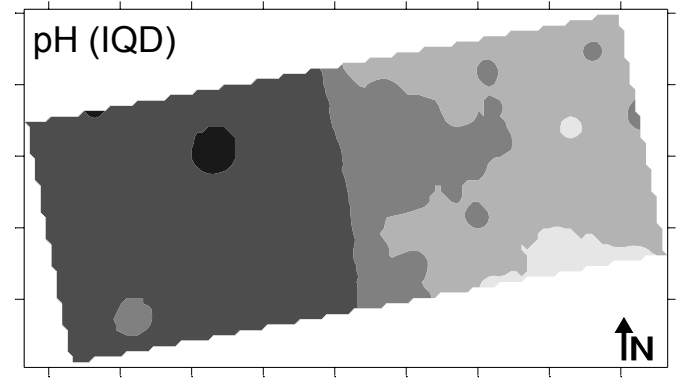

$\begin{array}{lllll}4.1 & 4.5 & 4.9 & 5.3 & 5.7\end{array}$

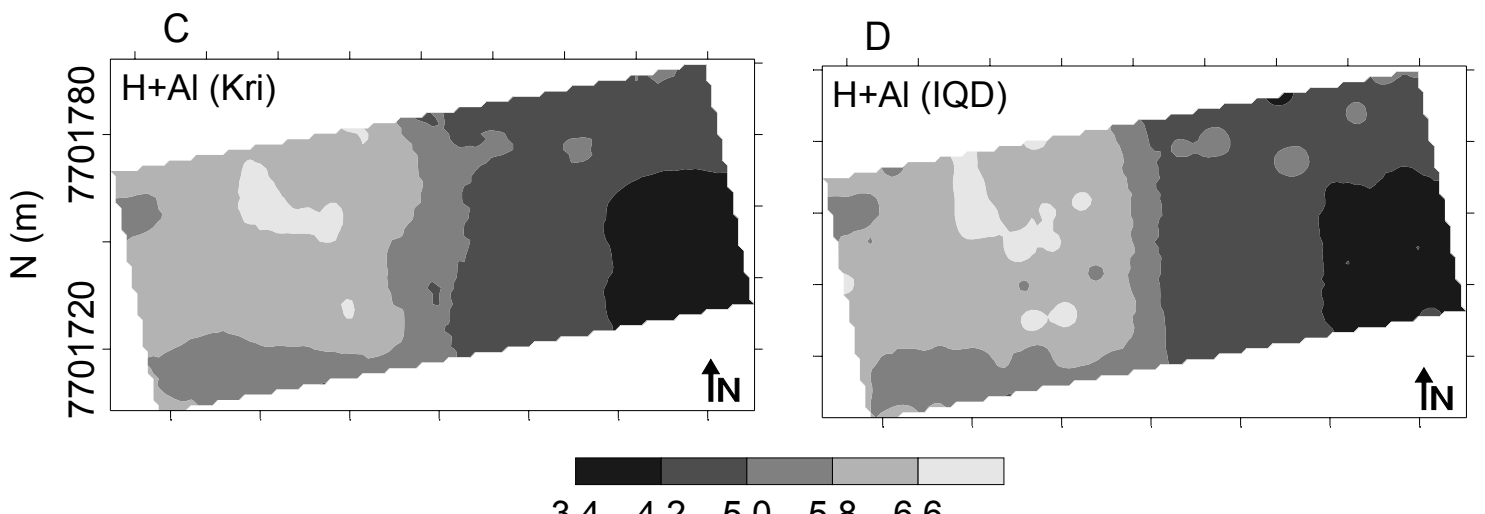

$\begin{array}{lllll}3.4 & 4.2 & 5.0 & 5.8 & 6.6\end{array}$

$\mathrm{E}$

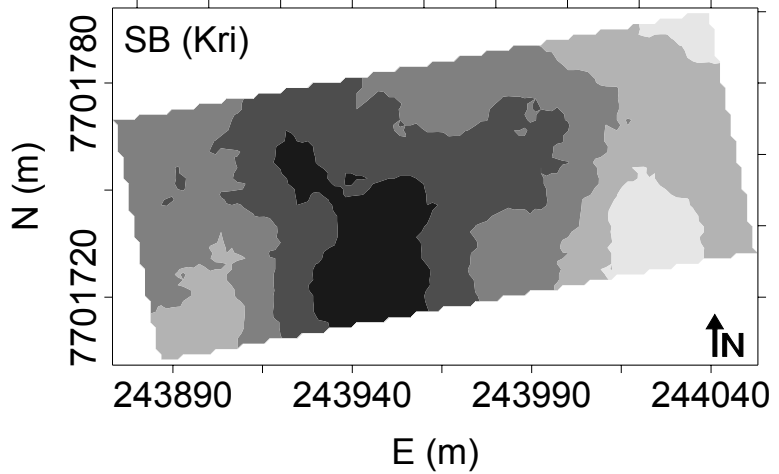

$\mathrm{F}$

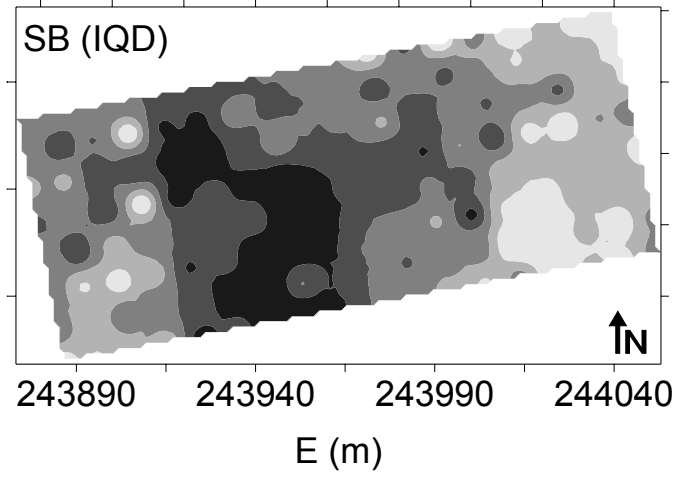

\section{$\begin{array}{lllll}0.6 & 1.0 & 1.4 & 1.8 & 2.2\end{array}$}

FIGURA 3 - Mapas de distribuição espacial dos atributos químicos do solo pH (A e B), H+Al (C e D) e soma de cátions básicos (SB) (E e F) interpolados respectivamente pela krigagem ordinária (Kri) e inverso do quadrado da distância (IQD).

Notou-se uma maior diferença visual entre os mapas interpolados por Kri e IQD para o atributo SB (Figuras 3E e 3F), apresentando o mapa interpolado por IQD uma maior variabilidade em relação à Kri, resultado da presença de uma menor uniformidade dos tons de cinza. Esse resultado é obtido principalmente pelo fato de que o interpolador Kri ser não-viciado e com variância mínima, sendo considerado um interpolador ótimo (Carvalho \& Assad, 2005).

Observou-se uma maior concentração dos maiores teores de SB na parte inferior e a direita da área, concordando com os maiores valores de $\mathrm{pH}$, ou seja, estes atributos apresentaram correlação $\left(r=0,63^{*}\right)$ significativa $(P<0,05) . \quad \mathrm{pH}$ do solo apresenta grande importância quanto a disponibilidade de nutrientes para a planta, entre eles o cálcio e o magnésio. Neste aspecto, a utilização de calcário dolomítico na área de pastagem contribuiu para elevar a disponibilidade dos cátions do solo, bem como elevar os teores de cálcio e magnésio, componentes da SB do solo. 
SOUZA, G.S. et al. Krigagem ordinária e inverso do quadrado...

O desempenho desses interpoladores foi comparado através da análise do coeficiente de determinação da validação cruzada $\left(R^{2} v c\right)$ e dos erros médios absoluto (EMA) e relativo (EMR), apresentados na Tabela 3 . Os dois métodos de interpolação foram eficientes na estimativa de valores para locais não amostrados apresentando $R^{2} v c$ significativo $(P<0,05)$ para os três atributos químicos em estudo.

TABELA 3 - Análise dos interpoladores krigagem ordinária (Kri) e inverso do quadrado da distância (IQD) na inferência de valores para os atributos químicos do solo $\mathrm{pH}, \mathrm{H}+\mathrm{Al}$ e soma de cátions básicos (SB).

\begin{tabular}{ccccc}
\hline Atributo & Interpolador & $\mathrm{R}^{2}{ }_{\mathrm{vc}}(\%)$ & EMA & EMR (\%) \\
\hline $\mathrm{pH}$ & Kri & $89,9^{* *}$ & $0,11 \mathrm{a}$ & $2,05 \mathrm{a}$ \\
& $\mathrm{IQD}$ & $89,7^{* *}$ & $0,12 \mathrm{a}$ & $2,06 \mathrm{a}$ \\
$\mathrm{H}+\mathrm{Al}$ & $\mathrm{Kri}$ & $85,1^{* *}$ & $0,29 \mathrm{a}$ & $5,62 \mathrm{a}$ \\
& $\mathrm{IQD}$ & $84,9^{* *}$ & $0,30 \mathrm{a}$ & $5,74 \mathrm{a}$ \\
$\mathrm{SB}$ & $\mathrm{Kri}$ & $38,4^{* *}$ & $0,38 \mathrm{a}$ & $27,12 \mathrm{a}$ \\
& $\mathrm{IQD}$ & $37,1^{* *}$ & $0,39 \mathrm{a}$ & $27,49 \mathrm{a}$ \\
\hline
\end{tabular}

** significativo $(\mathrm{P}<0,01)$. Médias seguidas pela mesma letra na coluna, para o mesmo atributo, não diferem significativamente pelo teste t-Student $(P<0,05)$.

A Kri apresentou um maior $R^{2} v c$ e os menores EMA e EMR para os três atributos químicos em estudo em relação ao IDQ. No entanto, observou-se similaridade entre os métodos de interpolação para os valores de $\mathrm{R}_{\mathrm{vc}}^{2}$, bem como diferença não significativa pelo teste t-Student $(P<0,05)$ para as estatísticas EMA e EMR.

Observou-se, para ambos os métodos de interpolação, menor valor de $R^{2}$ vc e maiores EMA e EMR para a SB em relação os demais atributos, visto que este atributo apresentou uma maior variabilidade, indicada pelo $\mathrm{CV}$, que resulta em estimativas com menor grau de confiabilidade em relação aos demais atributos.

Mello et al. (2003b) consideraram que a metodologia baseada no IQD pode apresentar imprecisões consideráveis, além de não ser o melhor método estatístico para a interpolação de parâmetros da equação de chuvas intensas, haja vista que a krigagem geoestatística pode promover melhorias consideráveis na precisão. Silva et al. (2008) encontraram maior erro na estimação dos dados com a interpolação com o inverso do quadrado da distância, entretanto sua diferença em relação à krigagem foi pequena, sendo os dois interpoladores considerados de boa precisão na inferência de valores para atributos do solo.

\section{CONCLUSÕES}

O $\mathrm{pH}, \mathrm{H}+\mathrm{Al}$ e $\mathrm{SB}$ apresentaram dependência espacial variando de forte a moderada, quantificada por meio do ajuste do modelo gaussiano aos semivariogramas experimentais, o que permitiu o uso do interpolador geoestatístico.

Os métodos de interpolação utilizados não apresentaram diferenças significativas para nenhum dos atributos químicos em estudo, no entanto observou-se diferença visual nos mapas interpolados pelos dois métodos, principalmente para a SB, indicando uma maior variabilidade do mapa gerado pelo inverso do quadrado da distância, o que pode resultar em maiores erros na estimativa.

A krigagem ordinária e o inverso do quadrado da distância foram eficientes na inferência de valores para locais não amostrados para os três atributos químicos analisados, indicado pela significância da validação cruzada, assim os dois metodos foram indicados para a espacialização de dados regionalizados referentes a atributos quimicos do solo.

\section{AGRADECIMENTOS}

Ao Conselho Nacional de Desenvolvimento Científico e Tecnológico do Brasil (CNPq) pela ajuda financeira, disponibilizando bolsa ao primeiro autor.

\section{REFERÊNCIAS}

1. ANGELICO, J. C.; SILVA, I. N. Eficiência da krigagem na estimativa das variáveis físicas e químicas do solo. Energia na Agricultura, v. 21, n. 1, p. 106-117, 2006

2. CARVALHO, J. R. P.; ASSAD, E. D. Análise espacial da precipitação pluviométrica no estado de São Paulo: comparação de métodos de interpolação. Engenharia Agrícola, v. 25, n. 2, p. 377-384, 2005.

3. CORÁ, J. E. et al. Variabilidade espacial de atributos do solo para adoção do sistema de agricultura de precisão na cultura de cana-de-açúcar. Revista Brasileira de Ciência do Solo, v. 28, n. 6, p. 1013-1021, 2004. 
4. COUTO, E. G.; SCARAMUZZA, J. F.; MARASCHINI, L. Influência dos métodos de interpolação dos dados nos mapas usados na agricultura de precisão. In: SIMPÓSIO INTERNACIONAL DE AGRICULTURA DE PRECISÃO, 2., 2002, Viçosa. Anais... Viçosa: SIAP, 2002. p. 1-5.

5. EMPRESA BRASILEIRA DE PESQUISA AGROPECUÁRIA (EMBRAPA). Centro Nacional de Pesquisa de Solos. Manual de métodos de análise do solo. 2. ed. Rio de Janeiro: Centro Nacional de Pesquisas de Solos, 1997. 212 p.

6. EMPRESA BRASILEIRA DE PESQUISA AGROPECUÁRIA (EMBRAPA). Sistema brasileiro de classificação de solos 2. ed. Rio de Janeiro: Centro Nacional de Pesquisas de Solos, 2006. $306 \mathrm{p}$

7. GOMES, N. M. et al. Variabilidade espacial de atributos físicos do solo associados ao uso e ocupação da paisagem. Revista Brasileira de Engenharia Agrícola e Ambiental, v. 11, n. 4, p. 427-435, 2007.

8. GREGO, C. R.; VIEIRA, S. R. Variabilidade espacial de propriedades físicas do solo em uma parcela experimental. Revista Brasileira de Ciência do Solo, v. 29, n. 2, p. 169-177, 2005.

9. KANEGAE JÚNIOR et al. Avaliação de interpoladores estatísticos e determinísticos como instrumento de estratificação de povoamentos clonais de Eucaliptus sp. Cerne, v. 12, n. 2, p. 123-136, 2006.

10.MELLO, C. R. et al. Krigagem e inverso do quadrado da distância para interpolação dos parâmetros da equação de chuvas intensas. Revista Brasileira de Ciência do Solo, v. 27, n. 5, p. 925-933, 2003a.

11.MELLO, C. R. et al. Modelos matemáticos para predição da chuva de projeto para regiões do Estado de Minas Gerais. Revista Brasileira de Engenharia Agrícola e Ambiental, v. 7, n. 1, p. 121-128, 2003b.

12.PREZOTTI, L. C. et al. Manual de recomendação de calagem e adubação para o estado do Espírito Santo. 5. aprox. Vitória: SEEA/INCAPER/CEDAGRO, 2007. 305 p.

13.ROBERTSON, G. P. GS+ Geostatistics for the environmental sciences: GS+ user's guide. Plainwell: Gamma Design Software, 1998. 152 p. 1 CD-ROM.

14.SILVA, S. A. et al. Avaliação de interpoladores estatísticos e determinísticos na estimativa de atributos do solo em agricultura de precisão. Idesia, v. 26, n. 2, p. 75-81, 2008.

15.SILVA, V. R. et al. Variabilidade espacial das características químicas do solo e produtividade de milho em um Argissolo Vermelho-Amarelo distrófico arênico. Revista Brasileira de Ciência do Solo, v. 27, n. 6, p. 1013-1020, 2003.

16.SOUSA, D. M. G. et al. Acidez do solo e sua correção. In: NOVAIS, R. F. et al. (Ed.). Fertilidade do solo. Viçosa: SBCS, 2007. p. 205-274.

17.SOUZA, G. S. et al. Variabilidade espacial do fósforo, potássio e da necessidade de calagem numa área sob pastagem. Revista Ciência Agronômica, v. 39, n. 3, p. 384-391, 2008a.

18. SOUZA, G. S. et al. Variabilidade espacial de atributos químicos em um Argissolo sob pastagem. Acta Scientiarum Agronomy, v. 30, n. 4, p. 589-596, 2008b.

19.SOUZA, Z. M.; MARQUES JUNIOR, J.; PEREIRA, G. T. Variabilidade espacial da estabilidade de agregados e matéria orgânica em solos de relevos diferentes. Pesquisa Agropecuária Brasileira, v. 39, n. 5, p. 491-499, 2004.

20.TIEPPO, R. C. et al. Avaliação de diferentes interpoladores na geração de mapas temáticos da produtividade de soja em agricultura de precisão. In: SIMPÓSIO INTERNACIONAL DE AGRICULTURA DE PRECISÃO, 4., 2007, Viçosa. Anais... Viçosa: UFV/CNPMS/SBEA, 2007. p. 1-4.

21.VIEIRA, S. R. Geoestatística em estudos de variabilidade espacial do solo. Tópicos em ciência do solo, v. 1, p. 1-54, 2000.

22.ZIMBACK, C. R. L. Análise espacial de atributos químicos de solos para fins de mapeamento da fertilidade. 2001 114 f. Tese (Livre-Docência em Levantamento do solo e fotopedologia) - Faculdade de Ciências Agronômicas, Universidade Estadual Paulista "Júlio de Mesquita Filho", Botucatu, 2001.

Recebido em 10/03/2009 Aceito em 09/09/2009 
